\title{
The Role of Physical Exercise for Menopausal Women
}

\author{
Ni Luh Kadek Alit Arsani \\ Faculty of Sport and Health \\ Universitas Pendidikan Ganesha \\ Singaraja, Bali, Indonesia \\ nicholebatch2@gmail.com
}

\author{
I Ketut Sudiana \\ Faculty of Sport and Health \\ Universitas Pendidikan Ganesha \\ Singaraja, Bali, Indonesia \\ sudiana_67@yahoo.co.id
}

\begin{abstract}
-menopause is a phase in a woman's life and many women are anxious and afraid of this phase. . according to a report from Badan Pusat Statistik (BPS) in 2013, the number of women aged 50 and above increased drastically from , 19.5 million in 2010 , to an estimated 39.5 million by the year 2030. With such large number and the average age for menopause being 51 years, it is evident that more than one third of a woman's life is spent after menopause. This will certainly cause health problems that would pose challenges for the medical sector especially in the area of trying to improve the health of women. Physical exercise plays an important role in improving the functions of women's reproductive organs .. Physical exercise improves ovulation rate and menstrual frequency, as well as restoring the functions of the reproductive organs through hormonal system repair. physical exercise increases the level of estrogen in individuals who exercises regularly unlike in those who do not exercise regularly..
\end{abstract}

\section{Keywords - physical exercise, menopausal women, health}

\section{INTRODUCTION}

Menopause is defined as the cessation of menstrual cycles in older women due to loss in ovarian activities. It is also known as amenorrhea. Another terminology that is associated with menopause is perimenopause. Perimenopause starts several years before the onset of menopause and ends after the onset of menopause. perimenopausal transition is defined as those years before the onset of menopause which include changes in the normal ovarian cycle to the cessation of menstruation , and this characterized by irregularities of the menstrual cycle [1].

An average Indonesian woman begins her menopause at the age of 50 though some of the women start at an earlier or later age. Physical factors and psychological factors influences menopause. Certain factors such as ovarian surgery, stress, medications, and lifestyle affect the rapid rate of menopause [2]. During menopause, a woman's reproductive capacity stops. The ovaries are no longer functioning, the production of steroid hormones and peptides gradually stops. Before the onset of menopause, in the late 30 s or early 40 s there is a sharp decline in the functional unit of the ovary and the follicles. This decrease in the number of follicles which are the main sources of ovarian hormones, causes irregularities of the hormonal system and disorganization of the hypothalamic-pituitary-ovarian axis. they also cause unpredictable endocrine status and various clinical abnormalities [3].

symptoms of menopause include: 1) general physical symptoms such as: hot flushes, night sweats, sleep disorders, fatigue, itching, bone pain caused by osteoporosis, headache, frequent urination, becoming fat with fat deposits around the waist and abdomen region, hair loss, wrinkled skin; 2) general psychic symptoms such as : anxiety, irritability, decreased concentration, decreased memory; 3) sexual symptoms which include: decrease in sexual drive, atrophy of vaginal epithelium, reduced vaginal lubrication when aroused which resulted in dyspareunia [2].

Physical exercise improves ones' physical fitness and provides health benefits. However, there are still many people who have sedentary behavioral patterns. In the United States, only $49.1 \%$ of the adult population does physical exercise recommended by the CDC-ASM physical exercise [4]. Therefore, this is major issue concerning the public health sector. The public health sector should create awareness about the health benefits of physical activities. . Physical exercise plays an important role in the improvement of women's reproductive functions. Physical exercise improves a woman's ovulation rate and menstrual frequency, as well as restore reproductive functions through hormonal system repair.

\section{HORMONAL CHANGES DUE TO MENOPAUSE}

The production of estrogen and progesterone is greatly reduced during menopause,. [ the reproductive system is a classical endocrine mode that is initiated by the pulsatile secretion of gonadotropin releasing hormones $(\mathrm{GnRH})$ from the hypothalamus to the pituitary gland via the portal vein system. GnRH regulates cystesis and the release of FSH and luteinizing hormone (LH) from the anterior pituitary gland.. FSH and LH stimulates ovarian follicle growth, ovulation, and corpus luteum formation and coordinates the secretion of estradiol, progesterone, inhibin A, and inhibin B. A key component of this system is the modulating effect of inhibin on gonadotropin secretion, which has an effect on the pituitary glands and also causes changes in the amplitude or frequency of the GnRH secretions. Control of the negative feedback on FSH secretion is essential for the development of a mature oocyte which is characteristic of the human reproductive cycle. In addition to the negative feedback control system, menstrual cycle is unique among other 
endocrine systems in terms of their dependence on the effect of positive estrogen feedback to that produces an essential preovulation LH surge for ovulation [5].

\section{A. Hormonal Changes in Perimenopausal Transition}

generally, perimenopausal changes are estimated by endocrine factors. Over the years, there has been a decrease in reproductive capacity. . this indicates a gametogenic failure of the ovary. Gametogenic failure is characterized by decreased inhibin secretion which occurs in the early follicular phase, increased serum FSH, and decreased fertility [6]. Longitudinal studies performed by SWAN (studies conducted on women in some countries) shows that estrogen levels begin to decline 2 years before the last menstrual period. Increased levels of FSH occur several years before menopause but increases distinctly 2 years before menopause and remain high until 2 years after menopause. There is also a gradual decline of androgens (androstenedione and testosterone) [6]. The number of oocytes decreases sharply and this begins to occur between the ages of 37-38 years. The mechanisms causing this decline is still very unclear, although there is this hypothesis that states that they may be associated with activin secretion. Activation derived from granulosa cells is important as it aids to stimulate the expression of FSH receptors [6]. This increase in FSH levels leads to an increase in activin production and the autocrine effect of this activation then improves the work of the FSH. This is expected to accelerate growth and differentiation of the granulosa cells, but at the same time there are many follicular atresia [6].

\section{B. Hormonal Changes in Postmenopausal Women}

The most significant changes are the decrease in estrogene levels E2 (E1) respectively. . Decreased serum E2 is greater than E1. Serum E1 is produced primarily from peripherals aromatization of androgens, which decrease more slowly. The average E2 levels is $15 \mathrm{pg} / \mathrm{mL}$ with a range between $10-25 \mathrm{pg} / \mathrm{mL}$. this might be lower than $10 \mathrm{pg} / \mathrm{ml}$ in women with oophorectomy.. The average level serum E1 is $30 \mathrm{pq} / \mathrm{mL}$ in obese women and may be higher because aromatization increases as the number of fat cells increases. [6]. The FSH levels increases 10-20 fold and the LH levels rises approximately 3 -fold until it reaches a maximum level of 1-3 years after menopause, and after that a gradual decrease occurs in both of these gonadotropins. Increased levels of FSH and $\mathrm{LH}$ in this period is an evidence of ovarian failure. FSH levels are higher than LH levels, this is because LH is cleansed faster than FSH in the blood (LH has a half-life of approximately 20 minutes and the half-life of FSH is 3-4 hours). the Decreased level of gonadotropin that occurs with age during postmenopausal is believed to be a reflection of the aging of the gonadotropin-secreting cells in the pituitary, which is as a result of a decreased ability to respond to gonadotropin releasing-hormone $(\mathrm{GnRH})$ [1].

After menopause, the ovaries primarily secrete androstenedione and testosterone, but there is decrease in the circulation of androstenedione after menopause. excess postmenopausal androstenedione is derived from the adrenal glands, and some are slightly derived from ovarian secretion, although androstenedione is a major steroid secreted by postmenopausal ovaries. Dehydroepiandrosterone (DHEA) and its sulfate (DHEAS), derived from the adrenal gland, decrease with age., ten years after menopause, the DHEA levels in circulation is $70 \%$ lower and $74 \%$ lower in younger females. [1]. Testosterone production decreases approximately by $25 \%$ after menopause, postmenopausal ovaries in most women secrete more testosterone than premenopausal ovaries, at least in the first few years of postmenopausal. the disappearance of follicules and estrogen, causes an increase in gonadotropin and this in turn causes the remaining ovary tissues to secrete more testosterone. However, the total amount of testosterone produced after menopause decreases as the primary source of testosterone also decreases.

In early postmenopausal, the circulating levels of androstenedione declines by approximately $62 \%$ in adulthood. In a longitudinal study conducted in Australia that had to do with observing menopausal effect 5 years before menopause and 7 years after menopause, it was deduced that there were no changes in the circulation of testosterone. . Obviously, this is because of the decrease in the sex hormone-binding globulin (SHBG), causing an increase in the free androgen. During the later years of postmenopausal, almost all androgen levels in circulation comes from the adrenal glands [1]. Finally, the steroidogenic tissue in the ovaries suffer damages, despite a large increase in FSH and LH levels,. Post-menopausal ovarian weight is less than 10 grams, and be detected through ultrasound, the adrenal contribution to the production of estrogen precursors decreases with age and this affects the, secondary sex tissues [1].

\section{PHYSICAL EXERCISE}

Physical exercises and physical activities are often used interchangeably., Physical activity is the movement of the body produced the contraction of the skeletal muscles which entails the use of energy when the body is not rest. While physical exercise is a form of physical activity that involves planned, structured, and repetitive body movements that are performed in order to enhance or maintain one or more of the physical fitness components. Physical fitness is specifically defined as a set of characteristics possessed or achieved by a person related to the ability to perform physical activities. [4]. to determine the range of intensity is an important guide of physical activities. . several methods are involved , : percentage of maximal oxygen comsumtion (VO2max), maximal heart rate (HRmax) or metabolic equivalents (METs) etc. Physical activities improves physical fitness and also provides health benefits. However, there are still many people who have sedentary behavior patterns. In the United States only $49.1 \%$ of the adult population performs physical activities as recommended by the CDC-ASM physical activity [4]. public health awaremess should be created in order to promote the health benefits of physical activities. 


\section{A. Guidelines of Physical Exercise for Health Benefits}

In relation to the health benefits gained by physical activity, the US annual Surgeon report came up with 2 important points: 1) health benefits can be achieved by moderate physical activities every day for 30 minutes and 2) additional health benefits can be gained by participating or engaging in more physical exercises. People who participate in physical activity programswith longer duration or with higher intensity regularly stands a chance to benefit more [4]. Sedentary behavioral patterns is still a habit for most people .., the American College of Sports Medicine (ACSM) and the American Heart Association (AHA) have recommend some good physical activities. [4].

1) The recommendations are as follows: (1) healthy adults between the ages of 18-65 should participate in moderate-intensity aerobic physical activities for 30 minutes 5 times in a week, adults with higher intensity should be engaged for 20 minutes 3 times in a week, (2) The combination of moderate and higher intensity is possible (3) Moderate-intensity aerobic physical activities can be achieved by doing a series of exercises for 10 minutes or more but at least a minimum of 30 minutes, (4) Every adult is adviced to engage in activities that maintain and improve strength and endurance at least twice a week.

2) The "FITT" guidelines provides maximum fitness with health benefits and minimal risks (e.g. cardiorespiratory disorders). The recommendations are as follows: (1) $\mathrm{F}=$ Frequency: 3 to 5 times a week, (2) I = Intensity: approximately $60-85 \%$ of maximal heart rate. This generally means that one exercises until he/she begins to sweat and breath heavily without any form of complaints (such as chest pain, dizziness). Maximum heart rate $=220$-age (in years) with a variation of 10 beats per minute, (2) $\mathrm{T}=$ Type (kind): a combination of aerobic exercise and calisthic activities .. Choice of activity on the basis of taste, state of fitness, availability of facilities and abilities, $(\mathrm{T})=$ Time (time): 15-60 minutes of continuous aerobic exercise. preceded by 3-5 minutes of warming up and followed by 3-5 minutes of relaxation.

exercise or workout programs for menopausal women should include, endurance exercises (aerobic), strength exercises, and balance exercises. of these exercises, aerobics, weight bearing, and resistance exercises are all effective in increasing the bone mineral density of the spine in postmenopausal women. Another effective exercise that should be prescribed is resistance and weight loss exercises and this should be done trice a week (on alternate days). To Warm up beforehand can help to reduce exercise related injuries and pain after exercising .. exercises or workout sessions should be done for at least two hours every week using 30 minutes for moderate aerobic activities. deep breathing, yoga, and stretching exercises can help to manage stress and menopause-related symptoms [7].

- Step 1: it is very important to warm up the body before any work out session. You could stretch, take a brisk walk or use a treadmill for five minutes ..
- Step 2: Engage in aerobic activities that increases the heart beat and burns fat. such as a dance class, aerobics class, running, cycling, kickboxing etc. these helps to develop the muscles and proper functioning of the cardiovascular system.

- Step 3: it is verycommon for women to experience osteoporosis during menopause. Weight lift exercises, use of resistant bands, strength trainings etc aids in keeping the bones and muscles strong. Strength training also aids in burning body fat, even while resting, to avoid the dreaded menopausal weight gain.

- Step 4: it also important to promote better flexibility by participating in workouts that causes the stretching of muscles, such as yoga and Pilates. This promotes better muscle functions. The woman must practice yoga and always meditate each night as this helps to reduce anxiety which is a common symptom of menopause.

- Step 5: Cool down at the end of every workout session by walking for a few minutes and stretching this helps to relieve any pain that may occur as a side effect from any particularly gruelling workout session. This gives the body a chance to relax and promotes regular breathing and reduction in heart beat. Thus, making the whole session a menopausal friendly workout session.

\section{B. Health Benefits of Physical Exercise for Menopausal Women}

Some researches have proven that physical exercise plays an important role in the improvement of the female reproductive organs.. Postmenopausal women who regularly exercise have significantly increased estrogen levels compared with those who do not perform any form of physical exercise [8]. Another research showed that physical exercise increases ovarian hormones. This indicates that the mechanisms associated with impaired ovarian function can be enhanced by physical exercises [9].

Exercises during and after menopause has many benefits:, [10].

- Preventing weight gain. Women tend to lose muscle mass and gain abdominal fat around menopause. Regular physical activity can help prevent weight gain.

- Reducing the risk of cancer. Exercise during and after menopause can help you lose excess weight or maintain a healthy weight, which might offer protection from various types of cancer, such as breast, colon and endometrial cancer.

- Strengthening bones. Exercise can slow bone loss after menopause, which lowers the risk of fractures and osteoporosis.

- Reducing the risk of other diseases. Menopause weight gain can have serious implications on your health. Excess weight increases the risk of heart disease and type 2 diabetes. Regular exercise can counter these risks. 
- Reducing the risk of other diseases. Menopausal weight gain can have serious implications on your health. Excess weight increases the risk of heart disease and type 2 diabetes. Regular exercise can counter these risks.

- Boosting mood. Physically active adults have a lower risk of depression and cognitive decline.

\section{CONCLUSIONS}

Regular exercise is recommended for menopausal women. This helps to sustain $\mathrm{s}$ their health. Exercise increases estrogen levels.. This can reduce clinical complaints that are often felt by menopausal women and long-term effects prevent osteoporosis thereby reducing the incidence of fractures in menopausal women. All this helps to improve the health of menopausal women.

\section{REFERENCES}

[1] A. M. Fritz, and L. Speroff, Clinical Gynecologic Endocrinology and Infertility. $8^{\text {th }}$. Ed. Philadelphia: Lippincott Williams \& Wilkins. 2011

[2] W. Pangkahila, Anti-Aging Tetap Muda dan Sehat. Jakarta: PT Kompas Media Nusantara. 2011.

[3] M. L. Batrinos, Premenopause: The Endocrinology of Reproductive Decline. Hormonos, 2013, vol 12(3), pp. 334-349.

[4] W. L. Haskell, I. M. Lee, R. R. Pate, "Physical Activity and Public Health: Updated Recommendation from the American College Of Sports Medicine And The American Heart Association," Med Sci Sports Exerc, vol 39(8), pp. 1423-34. 2007

[5] J. E. Hall, "Neuroendocrine Control of the Menstrual Cycle," In: J. F. Strauss, and R. Barbieri, editors. Yen \& Jaffe's Reproductive Endocrinology: Physiology, Pathophysiology, and Clinical Management. $7^{\text {th }}$. Ed. Philadelphia: Elsevier Saunders. pp. 141-156. 2014

[6] A. R. Lobo, "Menopause and Aging. In: J. F. Strauss, and R. Barbieri, editors. Yen \& Jaffe's Reproductive Endocrinology: Physiology, Pathophysiology, and Clinical Management," $7^{\text {th }}$. Ed. Philadelphia: Elsevier Saunders. pp. 308-339. 2014

[7] N. Mishra, V. N. Mishra, and Devanshi, "Exercise beyond menopause: Dos and Don'ts. J Midlife Health, vol 2(2), pp. 51-56, Jul-Dec 2011.

[8] V. Tarawan, , I. Akbar, A. Purba, F. Tandjung, "Correlation of serum estradiol, IL-6 and IGF-1 with bone density in menopausal women with and without regular exercise," Proceedings of The Physiological Society. $37^{\text {th }}$ Congress of IUPS (Birmingham, UK). 2013. Available from URL:
UPS: http://www.physoc.org/proceedings/abstract/Proc\%2037th\%20IUPSP CC250.

[9] I. J. Moran, C. L. Harrison, S. K. Hutchison, N. K. Stepto, B. J. Strauss, H. J. Teede, "Exercise Decreases Anti-Mullerian Hormone in Anovulatory Overweight Women with Polycystic Ovary Syndrome: A Pilot Study," Horm. Metab. Res, vol 43 (13), pp. 977-9. 2011

[10] M. Clinic, Fitness Tips for Menopause: Why Fitness Counts. Available from: https://www.mayoclinic.org/healthy-lifestyle/womens-health/indepth/fitness-tips-for-menopause/art-20044602. May 2016. 